\title{
REVISIONES DE ALCANCE, REVISIONES PARAGUAS Y SÍNTESIS ENFOCADA EN REVISIÓN DE MAPAS: ASPECTOS METODOLÓGICOS Y APLICACIONES
}

\author{
Diego Chambergo-Michilot@1,2,3,a, Mario E. Diaz-Barrera@4,5,a, Vicente A. Benites-Zapata@6, b \\ 1 Universidad Científica del Sur. Lima, Perú. \\ ${ }^{2}$ Department of Cardiology Research, Torres de Salud National Research Center, Lima, Peru. \\ 3 Red Latinoamericana de Cardiología, Lima, Perú. \\ ${ }^{4}$ Unidad de Investigación en Bibliometría, Universidad San Ignacio de Loyola, USIL. Lima, Perú. \\ ${ }^{5}$ Sociedad Científica de Estudiantes de Medicina de la Universidad Nacional de Trujillo, Trujillo, Perú. \\ ${ }^{6}$ Universidad San Ignacio de Loyola, Unidad para la Generación y Síntesis de Evidencias en Salud. Lima, Perú \\ a Estudiante de medicina humana; ${ }^{\text {b }}$ médico cirujano
}

\section{RESUMEN}

Nuestro objetivo fue comentar las diferencias metodológicas y aplicaciones de tres nuevos tipos de revisiones con especial atención a Latinoamérica. Entre estos nuevos tipos de revisiones tenemos a las revisiones de alcance, revisiones paraguas y síntesis enfocada en revisión de mapas. Las revisiones de alcance identifican la naturaleza y extensión de la evidencia, las revisiones paraguas se centran en la síntesis de todas las revisiones sistemáticas que abordan un mismo problema de salud, mientras que las síntesis enfocadas en la revisión de mapas son opciones válidas cuando resulta inviable un mapeo clásico de la evidencia, por abordar demasiados artículos. En la medida de que los investigadores en Latinoamérica se familiaricen con la metodología y utilidad de estos nuevos tipos de revisión, se observará un incremento de la producción científica.

Palabras clave: Medicina Basada en la Evidencia; Técnicas de Evaluación; Revisiones Sistemáticas; Metodología (Fuente: DeCS BIREME).

\section{SCOPING REVIEWS, UMBRELLA REVIEWS AND FOCUSED MAPPING REVIEW SYNTHESIS: METHODOLOGICAL ASPECTS AND APPLICATIONS}

\begin{abstract}
We aimed to comment on the methodological differences and applications of these three types of reviews with special attention to Latin America. Among these new types of reviews we have the scoping review, umbrella review and focused mapping review synthesis. Scoping reviews identify the nature and extent of the evidence, umbrella reviews focus on the synthesis of all systematic reviews that address the same health problem, while focused mapping review syntheses are valid options when a classical mapping of the evidence is not feasible, due to addressing too many articles. As researchers in Latin America become familiar with the methodology and usefulness of these new types of review, an increase in scientific production will be observed.
\end{abstract}

Keywords: Evidence Based; Medicine; Methods; Research Design; Systematic Review (Source: MeSH NLM)

Citar como: Chambergo-Michilot D, Diaz-Barrera ME, Benites-Zapata VA. Revisiones de alcance, revisiones paraguas y síntesis enfocada en revisión de mapas: aspectos metodológicos y aplicaciones. Rev Peru Med Exp Salud Publica 2021;38(1):136-42. doi: https://doi.org/10.17843/ rpmesp.2021.381.6501

Correspondencia: Vicente A. BenitesZapata; vbenites@usil.edu.pe

Recibido: $23 / 09 / 2020$

Aprobado: 27/01/2021

En línea: 24/02/2021

\section{INTRODUCCIÓN}

En la actualidad, la producción científica en los tópicos de ciencias de la salud evidencia un enorme y constante crecimiento ${ }^{(1,2)}$. En este escenario, los estudios que sintetizan la evidencia son muy útiles para responder diversas preguntas de investigación. La información generada por estos estudios es de utilidad para la práctica clínica y la toma de decisiones en salud pública. Entre todos los diseños de estudios de síntesis, el más conocido, es la revisión sistemática (RS). No obstante, existen otros tipos de revisiones, cada una con sus características, aportes metodológicos específicos y relevancia para las ciencias de la salud.

Un tipo de revisión que está ganando notoriedad en los últimos tiempos es la revisión de alcance [traducción en inglés: scoping review (SR)]. A diferencia de la RS, la SR permite evaluar un tema ampliamente. La pregunta de investigación es más general ${ }^{(3,4)}$, y la metodo- 
logía es ligeramente diferente a la RS ${ }^{(4,5)}$. Asimismo, la SR no se limita a identificar un solo diseño de estudios, sino que es posible incluir múltiples diseños. Entonces, la SR se hace relevante al permitir una exploración más vasta de la evidencia incluyendo todos los estudios que respondan la pregunta de investigación ${ }^{(6)}$. Por ejemplo, una SR que identifique las pruebas de diagnóstico más costo-efectivas en problemas reemergentes de salud pública ofrecería información relevante para el manejo político-sanitario ${ }^{(7)}$.

Existen otros tipos de revisiones que también son relevantes para la práctica clínica y la salud pública. La revisión paraguas [traducción en inglés: umbrella review (UR)] identifica múltiples artículos que sintetizaron la evidencia sobre un tema, es decir, una sinopsis de síntesis. La UR es útil para contrastar hallazgos de las RS o para ampliar la información sintetizada. Por ejemplo, Giannakou K y et al. ${ }^{\left({ }^{8}\right)}$, realizaron una UR sobre los meta-análisis que identificaron factores asociados a diabetes gestacional, concluyendo que la obesidad e hipotiroidismo fueron los factores que evidenciaron la asociación más consistente. Por otro lado, recientemente se ha descrito un nuevo tipo de revisión, las síntesis enfocada en revisión de mapas [traducción en inglés: focused mapping review synthesis (FMRS)], el cual mapea las características más importantes de un tópico desde un enfoque epistemológico ${ }^{(9)}$.

Comparado con el avance europeo y estadounidense, la realización de estos nuevos tipos de revisiones en Latinoamérica es mucho menor. La incorporación de SR, UR y FMRS suponen una gran ventaja para metodólogos, salubristas y clínicos, ya que otorgan una visión más compleja que complementa a la RS. Debido a esto, en esta revisión comentamos las diferencias metodológicas y aplicaciones de estos tres tipos de revisiones con especial atención a Latinoamérica.

\section{REVISIONES DE ALCANCE}

\section{Definición}

Una SR se define como una revisión que identifica rápidamente conceptos claves de un tema a través de una cobertura exhaustiva de la literatura ${ }^{(5)}$. Arksey H y O'Malley L, identificaron cuatro razones comunes para abordar una SR: 1) examinar la actividad científica, especialmente en campos donde es difícil observar la información disponible; 2) identificación rápida de la literatura previamente a una RS para conocer la factibilidad de realizar una; 3) sintetizar resultados científicos; e 4) identificar vacíos en la literatura ${ }^{(5)}$.

Usualmente, las SR abarcan preliminarmente la literatura, identificando la naturaleza y extensión de la evidencia ${ }^{(10)}$. La fortaleza de este tipo de revisión es que permite que los autores reporten una vasta y suficiente información sobre un tópico escogido. Tomando esto como premisa, una SR permite abordar más de una pregunta de investigación. Adicional- mente, existen vacíos donde la evidencia es muy limitada, por lo que una SR podrían identificar estudios de cualquier diseño para tratar de llenar ese vacío o generar nuevas hipótesis y recomendaciones. Algunas desventajas es que no produce síntesis cuantitativa, sino informativa, por lo que existe el peligro de que el lector tome las conclusiones como definitivas. Los lectores de las SR deben tomar las conclusiones como un reporte del estado actual de la evidencia.

\section{Aspectos metodológicos}

Los pasos metodológicos reportados por Arksey $\mathrm{H}$ y O’Malley Lun ${ }^{(5)}$ son 1) identificar la pregunta(s) de investigación; 2) identificar estudios relevantes; 3) seleccionar los estudios; 4) tabular los resultados; y 5) cotejar, resumir y reportar los datos. El Instituto Joanna Briggs actualizó la metodología en $2015^{(11)}$, sin embargo, se ha evidenciado que los usuarios de la Base de Datos de Revisiones Sistemáticas del Instituto Joanna Briggs indican que la metodología necesita mayor detalle en la descripción de criterios de inclusión y presentación de resultados ${ }^{(12)}$.

El grupo elaborador de PRISMA (Elementos de Informes Preferidos para Revisiones Sistemáticas y Metaanálisis, en español) reportó una lista de verificación. Esta se dividió en título, resumen, introducción, metodología, resultados, discusión y financiamiento. Destacamos que la metodología incluye registro del protocolo, criterios de elegibilidad, fuentes de búsqueda y valoración crítica de fuentes primarias en lugar de riesgo de sesgo, a diferencia de una RS ${ }^{(13)}$. Mientras que la RS está enfocada en obtener conclusiones en base a la síntesis de la evidencia, la SR informa el estado actual de la evidencia $^{(10)}$.

Los autores de esta revisión realizamos una tabla de síntesis sobre los tipos de revisión, incluyendo el SR (Tabla 1). Utilizamos la metodología SALSA (Search, AppraisaL, Synthesis and Analysis), que fue descrita por Grant y Booth ${ }^{(10)}$. Resumimos los tipos de revisión según su planteamiento de la idea (objetivos, registro de protocolo), búsqueda (criterios de elegibilidad, fuentes de información, selección de estudios), evaluación (extracción de datos, riesgo de sesgo), síntesis y análisis.

\section{Ejemplos de autores latinoamericanos}

Una SR puede apoyar la toma de decisiones informadas en el ámbito asistencial y de salud pública. Por ejemplo, Zammarchi y et al. ${ }^{(7)}$ elaboraron una SR en colaboración con investigadores de Italia y Perú. El objetivo principal fue realizar una revisión de la literatura para evaluar la rentabilidad del diagnóstico y las estrategias de tratamiento de la infección tuberculosa latente en migrantes. Para ello, recopilaron estudios que reportaron evaluación económica del tratamiento de tuberculosis latente indistintamente del diseño. Los autores incluyeron nueve estudios y concluyen que el cribado mediante interferón gamma es costo-efectivo en migrantes. 
Tabla 1. Metodología SALSA para ilustrar los tipos de revisiones de interés.

\begin{tabular}{|c|c|c|c|c|c|c|c|c|c|}
\hline \multirow{3}{*}{$\begin{array}{l}\text { Tipo de } \\
\text { revisión }\end{array}$} & \multirow{3}{*}{$\begin{array}{c}\begin{array}{c}\text { Planteamiento de } \\
\text { la idea }\end{array} \\
\text { Objetivos (ejemplos) }\end{array}$} & \multicolumn{8}{|c|}{ Metodología (SALSA) } \\
\hline & & \multicolumn{3}{|c|}{ Búsqueda } & \multicolumn{2}{|c|}{ Evaluación } & \multirow[b]{2}{*}{$\begin{array}{l}\text { Riesgo de } \\
\text { sesgo }\end{array}$} & \multirow[b]{2}{*}{ Síntesis } & \multirow[b]{2}{*}{ Análisis } \\
\hline & & $\begin{array}{l}\text { Registro de } \\
\text { protocolo }\end{array}$ & $\begin{array}{l}\text { Criterios de } \\
\text { elegibilidad }\end{array}$ & $\begin{array}{c}\text { Fuentes de } \\
\text { información }\end{array}$ & $\begin{array}{l}\text { Selección de } \\
\text { estudios }\end{array}$ & $\begin{array}{c}\text { Extracción de } \\
\text { datos }\end{array}$ & & & \\
\hline SR & $\begin{array}{c}\text { Transmisión de } \\
\text { dengue a receptores } \\
\text { de trasplantes }{ }^{(14)} \\
\\
\text { Rentabilidad de } \\
\text { la detección y el } \\
\text { tratamiento de la } \\
\text { infección tuberculosa } \\
\text { latente en inmigrantes }\end{array}$ & No & $\begin{array}{c}\text { Sin } \\
\text { restricciones a } \\
\text { tipos de estudio }\end{array}$ & $\begin{array}{l}\text { La mayor } \\
\text { cantidad } \\
\text { posible, pues } \\
\text { procura } \\
\text { recuperar } \\
\text { la mayor } \\
\text { cantidad } \\
\text { posible de } \\
\text { registros. }\end{array}$ & $\begin{array}{c}\text { Por duplicado e } \\
\text { independiente. } \\
\text { Ante } \\
\text { discrepancias } \\
\text { un tercer autor } \\
\text { entra como } \\
\text { dirimente. }\end{array}$ & $\begin{array}{l}\text { Por duplicado e } \\
\text { independiente } \\
\text { buscando } \\
\text { consensos } \\
\text { finales. Ante } \\
\text { discrepancias } \\
\text { un tercer autor } \\
\text { entra como } \\
\text { dirimente. }\end{array}$ & $\begin{array}{l}\text { Evaluación } \\
\text { formal no } \\
\text { necesaria, } \\
\text { salvo casos } \\
\text { específicos. }\end{array}$ & $\begin{array}{c}\text { Flujograma } \\
\text { PRISMA de } \\
\text { resultados incluidos. } \\
\\
\text { Características } \\
\text { generales de } \\
\text { los estudios } \\
\text { seleccionados y } \\
\text { descripción de } \\
\text { las variables del } \\
\text { problema según } \\
\text { el enfoque de } \\
\text { los diferentes } \\
\text { tipos de estudio } \\
\text { encontrados. }\end{array}$ & $\begin{array}{c}\text { Estado actual de las } \\
\text { investigaciones en } \\
\text { cuánto a un tema } \\
\text { determinado. } \\
\text { Precisar qué tipos } \\
\text { de estudios se han } \\
\text { conducido más y } \\
\text { qué vacíos existen. }\end{array}$ \\
\hline
\end{tabular}

\begin{tabular}{|c|c|c|c|c|c|c|c|}
\hline UR & $\begin{array}{l}\text { Efectos del ejercicio } \\
\text { físico en pacientes con } \\
\text { fibromialgia }{ }^{(22)} \\
\text { Diabetes mellitus y } \\
\text { complicaciones en } \\
\text { implantes dentales }{ }^{(24)}\end{array}$ & Sí & $\begin{array}{l}\text { Restringido } \\
\text { exclusivamente } \\
\text { a revisiones } \\
\text { sistemáticas. }\end{array}$ & $\begin{array}{c}\text { La mayor } \\
\text { cantidad } \\
\text { posible, pues } \\
\text { procura } \\
\text { recuperar } \\
\text { la mayor } \\
\text { cantidad } \\
\text { posible de } \\
\text { registros. }\end{array}$ & $\begin{array}{c}\text { Por duplicado e } \\
\text { independiente. } \\
\text { Ante } \\
\text { discrepancias } \\
\text { un tercer autor } \\
\text { entra como } \\
\text { dirimente. }\end{array}$ & $\begin{array}{l}\text { Por duplicado e } \\
\text { independiente } \\
\text { buscando } \\
\text { consensos } \\
\text { finales. Ante } \\
\text { discrepancias } \\
\text { un tercer autor } \\
\text { entra como } \\
\text { dirimente. }\end{array}$ & $\begin{array}{l}\text { Sí debe realizar } \\
\text { evaluación } \\
\text { formal usando } \\
\text { herramientas } \\
\text { estandarizadas. }\end{array}$ \\
\hline
\end{tabular}

$\begin{array}{cc}\begin{array}{c}\text { Flujograma } \\ \text { PRISMA de estudios } \\ \text { incluidos. }\end{array} & \begin{array}{c}\text { Evaluar la } \\ \text { concordancia de } \\ \text { los resultados } \\ \text { entre revisiones } \\ \text { sistemáticas de una } \\ \text { Características } \\ \text { menerales de las } \\ \text { investigación. }\end{array} \\ \begin{array}{c}\text { revisiones incluidas, } \\ \text { cantidad de estudios } \\ \text { por revisión, } \\ \text { factores evaluados } \\ \text { en cada estudio, } \\ \text { generalmente } \\ \text { agrupadas según } \\ \text { en la calidad } \\ \text { desenlace. }\end{array} & \begin{array}{c}\text { mevisiones includidas } \\ \text { al representar el }\end{array} \\ & \begin{array}{c}\text { máximo nivel de } \\ \text { evidencia sobre } \\ \text { el problema }\end{array} \\ & \text { abordado. }\end{array}$

Criterios de inclusión explícitos.

\begin{tabular}{|c|c|c|c|c|c|c|c|c|c|}
\hline \multirow[b]{2}{*}{ FMRS } & \multirow[b]{2}{*}{$\begin{array}{c}\text { Naturaleza y alcance } \\
\text { de los estudios de } \\
\text { violencia de género en } \\
\text { Europa }{ }^{(28)} \\
\text { Perfil de la } \\
\text { investigación } \\
\text { cualitativa en ciencias }^{\text {de la salud }}{ }^{(29)}\end{array}$} & \multirow[b]{2}{*}{ No } & \multirow[b]{2}{*}{$\begin{array}{l}\text { Restringido } \\
\text { al contenido } \\
\text { encontrado } \\
\text { en las revistas } \\
\text { y el periodo } \\
\text { seleccionados } \\
\text { que respondan } \\
\text { a la pregunta de } \\
\text { investigación. }\end{array}$} & \multirow[b]{2}{*}{$\begin{array}{l}\text { Selecciona } \\
\text { revistas } \\
\text { específicas } \\
\text { generalmente } \\
\text { según criterios } \\
\text { de impacto o } \\
\text { representativi- } \\
\text { dad del campo } \\
\text { a evaluar. }\end{array}$} & \multirow[b]{2}{*}{$\begin{array}{c}\text { Por } \\
\text { «calibración» de } \\
\text { todo el equipo } \\
\text { investigador. }\end{array}$} & \multirow[b]{2}{*}{$\begin{array}{c}\text { Por } \\
\text { «calibración» de } \\
\text { todo el equipo } \\
\text { investigador. }\end{array}$} & \multicolumn{3}{|c|}{ inclusión explícitos. } \\
\hline & & & & & & & $\begin{array}{l}\text { No se hace } \\
\text { una evaluación } \\
\text { formal, sino } \\
\text { una valoración } \\
\text { crítica por } \\
\text { todo el equipo } \\
\text { investigador. }\end{array}$ & $\begin{array}{c}\text { Características } \\
\text { temáticas de } \\
\text { las revistas } \\
\text { seleccionadas y de } \\
\text { los estudios que } \\
\text { cumplen criterios de } \\
\text { inclusión por cada } \\
\text { una de ellas. }\end{array}$ & $\begin{array}{l}\text { Sigue un enfoque } \\
\text { flexible con } \\
\text { énfasis en perfilar } \\
\text { un campo del } \\
\text { conocimiento en } \\
\text { lugar de sintetizar } \\
\text { la evidencia. }\end{array}$ \\
\hline
\end{tabular}

La presente tabla fue elaborada por los autores de la revisión.

SALSA: Search, Appraisal, Analysis y Synthesis.

SR: scoping review (revisión de alcance). UR: umbrella review (revisión de paraguas). FMRS: focused mapping review synthesis (síntesis enfocada en revisión de mapas).

Esta información es relevante a nivel de salud pública, dado que podría utilizarse para diseñar intervenciones similares contextualizadas al entorno local. Por otro lado, una SR es buena opción de síntesis cuando el tema es infrecuente. Por ejemplo, Cedano JA et al. con un equipo elaborador de Colombia, realizaron una SR que tenía por objetivo la revisión de literatura sobre la detección del dengue en donantes potenciales de órganos y el uso de estos órganos infectados. Los autores incluyeron tres estudios que cumplían sus criterios de elegibilidad, dos reportes de caso y una serie de casos. Los autores hicieron conclusiones sobre el tiempo de inicio de los síntomas, las manifestaciones clínicas y el tamizaje según área endémica ${ }^{(14)}$. Esta información será valiosa para investigadores que diseñarán futuros estudios más complejos sobre esta enfermedad o para infectólogos que realicen recomendaciones sobre la transmisión del dengue en receptores después de un trasplante.

\section{REVISIONES PARAGUAS}

\section{Definición}

La existencia de las UR responde a la abrumadora proliferación de RS, ya que se publican hasta 11 por día ${ }^{(15)}$. Las UR nacen de la necesidad de sintetizar todas las RS que abordan un mismo problema de salud. El manual de la Colaboración Cochrane reporta diferentes escenarios que justifican su realización: 1) diferentes intervenciones y un mismo desenlace; 2) misma intervención y mismo problema, pero diferentes re- 
sultados; 3) misma intervención, pero diferentes desenlaces; 4) identificación de efectos adversos; e 5) identificación de estudios no incluidos en revisiones previas sobre un tema ${ }^{(16)}$.

Dentro de las ventajas de la UR podemos destacar que compara y contrasta resultados de RS en torno a una pregunta específica, lo que permite una perspectiva general sobre la evidencia encontrada. Además, permite evaluar la consistencia de la evidencia alrededor de una pregunta (análisis de heterogeneidad) y las razones que la explicarían (análisis de sensibilidad) ${ }^{(17)}$. La principal desventaja es que identifica estudios de síntesis publicados previamente ${ }^{(18)}$, por lo que la calidad metodológica repercute en las conclusiones. Otra desventaja, es el riesgo de que varias de las RS incluidas hayan considerado los mismos estudios primarios dentro de su selección. Esta superposición de estudios, dependiendo de sus resultados, podría sobreestimar o infraestimar los resultados ${ }^{(17)}$. Entonces, este análisis tratando de diferenciar los estudios que se superponen podría ayudar a tener conclusiones menos sesgadas.

La principal utilidad de la UR es que es aprovechada por clínicos y tomadores de decisiones ${ }^{(18)}$, quienes, ante varias RS que responden a una misma pregunta, pueden tener una perspectiva general sobre la evidencia disponible, permitiendo tomar la mejor decisión en beneficio del paciente.

\section{Aspectos metodológicos}

Fusar-Poli ${ }^{(18)}$ propone 10 aspectos importantes a tener en cuenta en la elaboración de una UR. Se describen a continuación: 1) Asegurarse de que esté debidamente justificada. Este punto hace énfasis en que la pregunta debe ser altamente controversial o que esté afectada por sesgos que no han sido evaluados de manera sistemática. 2) Contar con un protocolo. 3) Definir claramente las variables de interés. 4) Estimar un indicador común del tamaño de efecto, lo que permite que las comparaciones sean más sencillas de interpretar. 5) Reportar adecuadamente la heterogeneidad y los sesgos potenciales. 6) Realizar adecuada clasificación de la evidencia. Los autores hacen una propuesta de cómo analizar la evidencia por cada estudio. Cabe destacar que esta no es la única opción, existiendo alternativas, como la metodología GRADE (Los Grados de Recomendación, Valoración, Desarrollo y Evaluación) ${ }^{(19)}$. 7)Realizar análisis de sensibilidad. Consiste en volver a analizar los resultados, separando a aquellos que se alejen de la distribución común. 8) Reportar transparentemente los resultados encontrados. 9) Usar un software de análisis adecuado. 10) Reconocer las limitaciones encontradas. En estudios de síntesis se debe considerar que su calidad está sujeta a la calidad de los estudios incluidos (Tabla 1).

\section{Ejemplos de autores latinoamericanos}

La base electrónica Scopus registró 19 artículos publicados desde el 2015 que tienen al menos un autor con filiación latinoamericana. Aunque no todos se adhieren a la metodología que hemos descrito, como De Sio et al. ${ }^{(20)}$ o el protocolo de Sanabria-Martinez et al. ${ }^{(21)}$, que incluyen otros tipos de estudios primarios a parte de las RS, la mayoría sí lo hace. Tal es el caso de Andrade et al. ${ }^{(22)}$ en Brasil, que describieron las características y la calidad de las revisiones publicadas sobre los efectos del ejercicio físico en pacientes con fibromialgia. Los autores solo incluyeron RS (independientemente del metaanálisis). También en Brasil, Siqueira et al. ${ }^{(23)}$ describieron la calidad de la evidencia sobre el efecto del ejercicio físico en pacientes con hemofilia. Los autores incluyeron 10 RS. En otro caso, Mauricio et al. ${ }^{(24)}$ resumieron la evidencia sobre las asociaciones entre diabetes mellitus y complicaciones en implantes dentales. Los autores incluyeron 20 RS y ningún estudio fue de alta calidad metodológica.

Tal como se ha descrito, estos estudios se caracterizaron por realizar sistemáticamente la búsqueda, presentación de resultados, evaluación de calidad metodológica y el reporte de superposición de los estudios primarios incluidos en las revisiones.

\section{SÍNTESIS ENFOCADA EN REVISIÓN DE MAPAS}

\section{Definición}

Esta nueva propuesta, fue presentada por Bradbury-Jones et al. ${ }^{(9)}$ quienes la definen desde cuatro características principales, estas son 1) el enfoque en un campo definido del conocimiento en lugar del cuerpo de la evidencia; 2) mapeo de las características principales de la investigación de ese campo en lugar de sintetizar el contenido; 3) comenta el enfoque general de la producción científica en lugar del estado de la evidencia; y 4) examina desde un enfoque epistemológico. La FMRS resulta útil para preguntas en las que el mapeo clásico (síntesis enfocada en revisión de mapas) resultaría inviable por abordar demasiados artículos ${ }^{(9)}$. También son útiles por que la flexibilidad de su diseño permite responder una variedad de preguntas puntuales que surgen de áreas del conocimiento complejas.

Dentro de sus principales ventajas está el identificar las características de un campo de investigación que puede ser teórico, epistemológico o metodológico, procurando aportar comentarios críticos sobre sus aplicaciones y limitaciones. Por otro lado, la principal limitación es que el análisis es muy dependiente de la experiencia del equipo elaborador. Esto va a depender tanto de la familiaridad con el tema y el problema, así como su capacidad de conceptualizar parámetros que respondan a la pregunta de investigación desde el inicio de la revisión. Definir los límites del enfoque inicial en este tipo de revisiones puede ser un completo desafío, por lo que es necesario un liderazgo multidisciplinario, colaborador y adaptativo ${ }^{(9)}$.

\section{Aspectos metodológicos}

El proceso de elaboración de las FMRS se podría explicar a través de tres etapas: enfoque, mapeo y síntesis ${ }^{(9)}$. Cada una de estas incluye fases específicas. Es preciso recalcar que el objetivo no es revisar extensamente la literatura ni evaluarla críticamente, por el contrario, se centra principalmente en 
describir; ya sea la producción en un periodo previamente determinado o mostrar el perfil de la investigación actual en un área determinada ${ }^{(9)}$. Las etapas son las siguientes:

1) Enfoque. Aquí se especificarán los dos criterios principales, escoger las revistas que serán usadas como fuentes de datos y qué periodos de evaluación serán tomados en cuenta. Todo parte del desarrollo de una pregunta de investigación bien definida que permita desarrollar criterios de elegibilidad claros. A este proceso de consenso, se le conoce como calibración, y es en el cual los autores pueden definir el alcance de la investigación, la identificación de las revistas a considerar como fuentes de información y el periodo que analizarán de estas. Para esta fase se suelen apoyar realizando un SR para identificar previamente el contenido relevante al que quieran llegar. Así mismo, es importante tomar en cuenta que una cantidad insuficiente de fuentes de información o un margen de tiempo muy estrecho, podría representar una limitación al responder a la pregunta. Esta etapa finaliza cuando luego del consenso, se procede a la búsqueda y selección de información ${ }^{(9)}$.

2) Mapeo. La finalidad de esta etapa es presentar cualitativamente los datos encontrados, de tal forma que permita visualizar superficialmente, áreas de potencial interés para futuras investigaciones ${ }^{(25)}$. A diferencia de los mapeos de la evidencia clásicos, se desarrolla un proceso que permite un enfoque más específico. En esta etapa se elabora una matriz de extracción de datos (Bradbury-Jones et al. ${ }^{(9)}$ proponen un modelo de referencia) y a partir de esta, se conduce la presentación de mapas gráficos o diagramáticos, según las revistas fuente o según preguntas de investigación.

3) Síntesis. El objetivo de esta etapa no es sintetizar resultados de los estudios individuales, sino perfilar un campo de conocimiento, a lo cual están orientados los procesos de síntesis de esta etapa. Se puede añadir la evaluación de calidad si resultara necesario para responder las preguntas planteadas ${ }^{(9)}$ (Tabla 1).

\section{Ejemplos latinoamericanos}

No se encontraron estudios de autores latinoamericanos. Sin embargo, en 2016, Taylor et al. ${ }^{(26)}$ realizaron una FMRS para perfilar la situación de la traumatización vicaria, aquella que se produce de manera indirecta en una persona al estar expuestas a detalles de situaciones traumáticas ${ }^{(27)}$. Se propuso un marco conceptual que refleja el grado con que las investigaciones tratan temas que pueden causar malestar o angustia con la forma en que han sido capturados los datos en términos de su cercanía a los participantes. Otro ejemplo, es el de Bradbury-Jones et al. ${ }^{(28)}$, quienes procuraron crear un perfil de la naturaleza y el alcance de los estudios de violencia de género en Europa. Los resultados demuestran que la violencia de pareja y abuso sexual como temas de investigación son aquellos que más atraen la atención. Por último, otro trabajo obtuvo un perfil del estado de la investigación cualitativa en salud o ciencias sociales. De los 102 artículos seleccionados, 39 no describen el enfoque cualitativo en el título, 17 lo describen explícitamente, y 46 lo sugerían implícitamente. Los autores desarrollaron un recurso visual para ayudar a los investigadores cualitativos, con énfasis en los novatos, a localizar la orientación y las técnicas de sus estudios ${ }^{(29)}$.

\section{DISCUSIÓN}

Realizamos una revisión narrativa sobre tres tipos de revisiones diferentes a la RS. La aplicación del SR consta en cubrir exhaustivamente la evidencia sobre un tópico, abordan diferentes preguntas de investigación sobre un mismo tema, y pueden sugerir vacíos, así como nuevas hipótesis. La UR identifica revisiones que respondan una misma pregunta, permitiendo evaluar la consistencia de la evidencia. La novedad del FMRS consta en que genera un mapa descriptivo de las características más importantes de un área mediante un enfoque epistemológico.

Actualmente no se han publicado estudios que registren la producción científica general de cada continente. Los ejemplos que hemos utilizado en esta revisión fueron elaborados por investigadores de Perú, Brasil y Colombia. Actualmente, la producción científica en estudios de síntesis por autores con afiliación latinoamericana es incipiente. Probablemente esta falta de producción científica se deba al desconocimiento de la metodología de estos tipos de revisiones. En ese sentido, se justifica la elaboración de este artículo como una herramienta de difusión de estos diseños.

Recientemente en países latinoamericanos, como Perú y Colombia, se vienen realizando las revisiones/síntesis rápidas para la toma de decisiones. Estas síntesis rápidas responden a la necesidad de respuestas inmediatas ante problemas en salud pública que ameritan soluciones prontas. En estas revisiones se incluyen todos los estudios que le correspondan a su pregunta, de forma similar a las SR. Por ejemplo, investigadores del Instituto Nacional de Salud del Perú desarrollaron una revisión rápida sobre el uso de mascarillas y respiradores para la prevención y control de infecciones por virus respiratorios, incluyendo revisiones sistemáticas, ensayos clínicos y estudios observacionales ${ }^{(30)}$. Estos estudios son más rápidos que una RS pero la evaluación crítica es menos rigurosa, y las inferencias están limitadas en responder las preguntas ${ }^{(31)}$. Cada vez las revisiones rápidas van ganando terreno en la toma decisiones en salud pública, sobre todo para principalmente conocer las intervenciones más costo-efectivas ${ }^{(32)}$.

Otro punto importante donde podrían ayudar la SR es la elaboración de guías de práctica clínica (GPC). En los últimos 
años, se ha reportado un esfuerzo por mejorar la calidad de las GPC a través del uso de una metodología internacionalmente aceptada, la GRADE, especialmente en Colombia y Perú ${ }^{(33)}$. Esta metodología recalca la importancia de incluir RS como fuente para generar recomendaciones en las GPC ${ }^{(19)}$. Entonces, la implementación formal de los SR como fuente de información adicional supondría una ventaja.

En cuanto a las UR, existen autores que proponen metodologías contrapuestas a lo descrito, en las que afirman que los ensayos clínicos también son estudios que deben incluirse en la búsqueda sistemática de UR ${ }^{(34)}$. Sin embargo, existe otro importante grupo de autores quienes se ajustan a lo descrito. Es preciso recalcar algunas diferencias con los meta-análisis en red, los cuales tienen por objetivo la comparación de múltiples intervenciones de manera simultánea en un mismo análisis cuantitativo, combinando de manera directa e indirecta evidencia dentro de una red de ensayos clínicos ${ }^{(35)}$, mientras que las UR incluyen RS que evalúan una misma pregunta clínica, de esa forma presentan sus datos cualitativamente, y enfatizan en la calidad metodológica, siendo su fin principal el análisis del máximo nivel de evidencia.

Respecto a las FMRS, encontramos similitudes con los análisis bibliométricos dado que evalúan la actividad en un tema o campo científico. Sin embargo, los estudios bibliométricos utilizan métodos estadísticos para conocer el impacto científico de los artículos, las redes de colaboración, frecuencia de tipos de estudios publicados, tópicos, posición en el cuartil de impacto, entre otras, de un autor, institución o país/ región ${ }^{(36)}$. En cambio, la FMRS tiene un enfoque más cualitativo tratando de organizar los estudios mediante un marco conceptual. Además, estos estudios generan herramientas gráficas que resumen los hallazgos y son fáciles de interpretar.

Por otro lado, las FMRS se podrían confundir con las revisiones de mapeado (traducción en inglés: mapping reviews), porque caracterizan el conocimiento sobre el tópico, describiendo teorías, nuevos hallazgos, metodologías, entre otras ${ }^{(37)}$. Sin embargo, no se elabora un marco conceptual con los estudios encontrados ni tampoco se producen herramientas gráficas para resumir el conocimiento. Por ejemplo, Langøien LJ et al. ${ }^{(38)}$ elaboraron una revisión de mapeado sobre los factores asociados con actividad física y sedentarismos entre los grupos étnicos minoritarios en Europa. Los autores agruparon los 165 factores observados en ocho conglomerados, como ambiente sociocultural, contexto migratorio, ambiente político, entre otros, y discutieron los hallazgos.

Mediante este artículo pretendemos aumentar el entendimiento y difusión de los usos de los tipos de revisión diferentes a la RS para el contexto latinoamericano. Recomendamos este tipo de estudios cuando se quieran revisar el estado actual de la evidencia sobre un tema, evaluar intervenciones farmacológicas y no-farmacológicas para elaborar políticas sanitarias o entender y organizar la actividad de la investigación sobre algún tópico. Cada uno de los tipos de revisiones tiene ventajas y desventajas, por lo que se debe estudiar exhaustivamente la metodología antes de elaborarlas (Tabla 1). Finalmente, recomendamos la valoración de la enseñanza de estas metodologías en los currículos de pregrado, ya que es de utilidad en el marco teórico de las tesis, y para publicar artículos sobre el estado de arte de un tópico.

\section{CONCLUSIONES}

Existen nuevos tipos de estudios de síntesis que comparten similitudes y diferencias metodológicas con las RS. Estos estudios son útiles en el ámbito de la práctica clínica y la salud pública. Solo pudimos encontrar ejemplos con autores latinoamericanos en SR y UR. En la medida de que los investigadores en Latinoamérica se familiaricen con la metodología y utilidad de estos nuevos tipos de revisión, se observará un incremento de la producción científica.

Contribuciones de autoría: DCM y MD participaron en la redacción del primer borrador, interpretación de los resultados, revisión crítica del manuscrito y revisión de la versión final VABZ participó en la concepción del estudio, diseño del estudio, interpretación de resultados, revisión crítica del manuscrito y revisión de la versión final

Financiamiento: Autofinanciado.

Conflictos de interés: Los autores declaran no tener conflictos de interés.

\section{REFERENCIAS BIBLIOGRÁFICAS}

1. Khademloo M, Khaseh AA, Siamian H, Aligolbandi K, Latifi M, Yaminfirooz M. Study of Scientific Production of Community Medicines' Department Indexed in ISI Citation Databases. Acta Inform Med. 2016;24(5):370-4. doi: 10.5455/aim.2016.24.370-374.

2. Sambo M do R, Ferreira AVL. Current status on health sciences research productivity pertaining to Angola up to 2014. Health Res Policy Syst. 2015;13. doi: 10.1186/s12961-015-0021-z.

3. Moher D, Stewart L, Shekelle P. All in the Family: systematic reviews, rapid reviews, scoping reviews, realist reviews, and more. Systematic Reviews. 2015;4(1):183. doi: 10.1186/s13643-015-0163-7.
4. Peters MDJ, Godfrey CM, Khalil H, McInerney P, Parker D, Soares CB. Guidance for conducting systematic scoping reviews. JBI Evidence Implementation. 2015;13(3):141-146. doi: 10.1097/ XEB. 0000000000000050

5. Arksey H, O’Malley L. Scoping studies: towards a methodological framework. International Journal of Social Research Methodology. 2005;8(1):19-32. doi: 10.1080/1364557032000119616.

6. Peterson J, Pearce PF, Ferguson LA, Langford CA. Understanding scoping reviews: Definition, purpose, and process. J Am Assoc Nurse Pract. 2017;29(1):12-6. doi: 10.1002/2327-6924.12380. 
7. Zammarchi L, Casadei G, Strohmeyer M, Bartalesi F, Liendo C, Matteelli A, et al. A scoping review of cost-effectiveness of screening and treatment for latent tubercolosis infection in migrants from high-incidence countries. BMC Health Serv Res. 2015;15:412. doi: 10.1186/s12913-015-1045-3.

8. Giannakou K, Evangelou E, Yiallouros P, Christophi CA, Middleton $\mathrm{N}$, Papatheodorou E, et al. Risk factors for gestational diabetes: An umbrella review of meta-analyses of observational studies. PLoS ONE. 2019;14(4):e0215372. doi: 10.1371/journal.pone.0215372.

9. Bradbury-Jones C, Breckenridge JP, Clark MT, Herber OR, Jones C, Taylor J. Advancing the science of literature reviewing in social research: the focused mapping review and synthesis. International Journal of Social Research Methodology. 2019;22(5):451-62. doi: 10.1080/13645579.2019.1576328.

10. Grant MJ, Booth A. A typology of reviews: an analysis of 14 review types and associated methodologies. Health Information \& Libraries Journal. 2009;26(2):91-108. doi: 10.1111/j.1471-1842.2009.00848.x.

11. Joanna Briggs Institute. The Joanna Briggs Institute Reviewers' Manual 2015: Methodology for JBI Scoping Reviews. Adelaide, Austra- lia: Joanna Briggs Institute; 2015. Accessed at http://joannabriggs.org/assets/docs/sumari/ Reviewers-Manual_Methodology-for-JBI-Scoping-Reviews_2015_v2.pdf on 3 August 2018 .

12. Khalil H, Bennett M, Godfrey C, McInerney P, Munn Z, Peters M. Evaluation of the JBI scoping reviews methodology by current users. Int J Evid Based Healthc. 2020;18(1):95-100. doi: 10.1097/XEB.0000000000000202

13. Tricco AC, Lillie E, Zarin W, O’Brien KK, Colquhoun $\mathrm{H}$, Levac D, et al. PRISMA Extension for Scoping Reviews (PRISMA-ScR): Checklist and Explanation. Ann Intern Med. 2018;169(7):467-73. doi: 10.7326/M18-0850.

14. Cedano JA, Mora BL, Parra-Lara LG, Manzano-Nuñez R, Rosso F. A scoping review of transmission of dengue virus from donors to recipients after solid organ transplantation. Trans R Soc Trop Med Hyg. 2019;113(8):431-6. doi: 10.1093/trstmh/trz024.

15. Bastian H, Glasziou P, Chalmers I. Seventy-Five Trials and Eleven Systematic Reviews a Day: How Will We Ever Keep Up?. PLOS Medicine. 2010;7(9):e1000326. doi: 10.1371/journal.pmed.1000326.

16. Higgins JPT, Thomas J, Chandler J, Cumpston M, Li T, Page MJ, Welch VA, et al (editors). Cochrane Handbook for Systematic Reviews of Interventions version 6.0 (updated July 2019). Cochrane, 2019. Available from www. training.cochrane.org/handbook.

17. Aromataris E, Fernandez R, Godfrey CM, Holly C, Khalil H, Tungpunkom P. Summarizing systematic reviews: methodological development, conduct and reporting of an umbrella review approach. Int J Evid Based Healthc. 2015;13(3):132-40. doi: 10.1097/XEB.0000000000000055.

18. Fusar-Poli P, Radua J. Ten simple rules for conducting umbrella reviews. Evidence-Based Mental Health. 2018;21(3):95-100. doi: 10.1136/ebmental-2018-300014.

19. Guyatt G, Oxman AD, Akl EA, Kunz R, Vist G, Brozek J, et al. GRADE guidelines: 1. Introduction-GRADE evidence profiles and summary of findings tables. J Clin Epidemiol. 2011;64(4):383-94. doi: 10.1016/j.jclinepi.2010.04.026.

20. De Sio S, Buomprisco G, Perri R, Bruno G, Mucci N, Nieto HA, et al. Work-related stress risk and preventive measures of mental disorders in the medical environment: an umbrella review. Eur Rev Med Pharmacol Sci. 2020;24(2):821-30. doi: 10.26355/eurrev_202001_20065.

21. Sanabria-Martínez G, Poyatos-León R, Notario-Pacheco B, Álvarez-Bueno C, Cavero-Redondo I, Martinez-Vizcaino V. Effects of physical exercise during pregnancy on mothers' and neonates' health: a protocol for an umbrella review of systematic reviews and meta-analysis of randomised controlled trials. BMJ Open. 2019;9(9). doi: 10.1136/bmjopen-2019-030162

22. Andrade A, Dominski FH, Sieczkowska SM. What we already know about the effects of exercise in patients with fibromyalgia: An umbrella review. Seminars in Arthritis and Rheumatism. 2020. doi: 10.1016/j. semarthrit.2020.02.003.

23. Siqueira TC, Dominski FH, Andrade A. Effects of exercise in people with haemophilia: An umbrella review of systematic reviews and meta-analyses. Haemophilia. 2019;25(6):928-37. doi: 10.1111/hae.13868.
24. Meza Maurício J, Miranda TS, Almeida ML, Silva HD, Figueiredo LC, Duarte PM. An umbrella review on the effects of diabetes on implant failure and peri-implant diseases. Braz Oral Res. 2019;33(suppl 1):e070. doi: 10.1590/1807-3107bor-2019.vol33.0070.

25. Davidson E, Edwards R, Jamieson L, Weller S. Big data, qualitative style: a breadth-and-depth method for working with large amounts of secondary qualitative data. Qual Quant. 2019;53(1):363-76. doi: 10.1007/s11135-0180757-y.

26. Taylor J, Bradbury-Jones C, Breckenridge JP, Jones C, Herber OR. Risk of vicarious trauma in nursing research: a focused mapping review and synthesis. J Clin Nurs. 2016;25(19-20):2768-77. doi: 10.1111/jocn.13235.

27. Lerias D, Byrne MK. Vicarious traumatization: symptoms and predictors. Stress and Health. 2003;19(3):129-38. doi: 10.1002/smi.969.

28. Bradbury-Jones C, Appleton JV, Clark M, Paavilainen E. A Profile of Gender-Based Violence Research in Europe: Findings From a Focused Mapping Review and Synthesis. Trauma Violence Abuse. 2019;20(4):470-83. doi: $10.1177 / 1524838017719234$.

29. Bradbury-Jones C, Breckenridge J, Clark MT, Herber OR, Wagstaff C, Taylor J. The state of qualitative research in health and social science literature: a focused mapping review and synthesis. International Journal of Social Research Methodology. 2017;20(6):627-45. doi: 10.1080/13645579.2016.1270583.

30. Instituto Nacional deSalud(Perú). Tratamiento específico parala infección por COVID-19. Elaborado por Karen Huamán, CatherineBonilla, Fabiola Huaroto, Maricela Curisinche, Nora Reyes, Ericson Gutierrezy Patricia Caballero. Lima: Unidad de Análisis y Generación de Evidencias en Salud Pública. Instituto Nacional de Salud, abril de 2020. Serie Revisión Rápida No 05-2020.

31. Universidad De Antioquia. Intervenciones no farmacológicas para la contención, mitigación y supresión de la infección por COVID-19. Colombia: Antioquia; 2020. Disponible en: https://es.cochrane.org/sites/es.cochrane. org/files/public/uploads/COVID-19/udea-uned_sintesisrapida_covid19_ ncov19_nofarmacologicas_rapidsynthesis_covid19_ncov19_nonpharmacological_28mar2020.pdf.

32. Lessa F, Caccavo F, Curtis S, Ouimet-Rathé S, Lemgruber A. Strengthening and implementing health technology assessment and the decision-making process in the Region of the Americas. Rev Panam Salud Publica. 2017;41. doi: 10.26633/RPSP.2017.165.

33. Cabrera PA, Pardo R. Review of evidence based clinical practice guidelines developed in Latin America and Caribbean during the last decade: an analysis of the methods for grading quality of evidence and topic prioritization. Globalization and Health. 2019;15(1):14. doi: 10.1186/s12992-019-0455-0.

34. Tsagris M, Fragkos KC. Umbrella Reviews, Overviews of Reviews, and Meta-epidemiologic Studies: Similarities and Differences. In: Biondi-Zoccai G, editor. Umbrella Reviews: Evidence Synthesis with Overviews of Reviews and Meta-Epidemiologic Studies. Cham: Springer International Publishing; 2016. p. 43-54. doi: 10.1007/978-3-319-25655-9_4.

35. Rouse B, Chaimani A, Li T. Network meta-analysis: an introduction for clinicians. Intern Emerg Med. 2017;12(1):103-11. doi: 10.1007/s11739016-1583-7.

36. Agarwal A, Durairajanayagam D, Tatagari S, Esteves SC, Harlev A, Henkel $\mathrm{R}$, et al. Bibliometrics: tracking research impact by selecting the appropriate metrics. Asian J Androl. 2016;18(2):296-309. doi: 10.4103/1008-682X.171582.

37. Paré G, Kitsiou S. Chapter 9 Methods for Literature Reviews. Handbook of eHealth Evaluation: An Evidence-based Approach [Internet]. University of Victoria; 2017.

38. Langøien LJ, Terragni L, Rugseth G, Nicolaou M, Holdsworth M, Stronks $\mathrm{K}$, et al. Systematic mapping review of the factors influencing physical activity and sedentary behaviour in ethnic minority groups in Europe: a DEDIPAC study. Int J Behav Nutr Phys Act. 2017;14(1):99. doi: 10.1186/ s12966-017-0554-3. 